\title{
Dynamic Neural Network Based Nonlinear Control of a Distillation Column
}

\author{
Feng $\mathbf{L i}^{1,2}$ \\ ${ }^{1}$ Department of Mathematics and Statistics, University of Maryland, Baltimore, USA \\ ${ }^{2}$ Office of Biostatistics, Center for Drug Evaluation and Research, \\ Food and Drug Administration, Silver Spring, USA \\ E-mail:fengli@fda.hhs.gov \\ Received July 27, 2011; revised August 19, 2011; accepted August 26, 2011
}

\begin{abstract}
Taking advantage of the knowledge of top and bottom compositions of a distillation column, a dynamic neural network (DNN) is designed to identify the input-output relationship of the column. The weight-training algorithm is derived from a Lyapunov function. Based on this empirical model, a nonlinear $\mathrm{H}_{\infty}$ controller is synthesized. The effectiveness of the control strategy is demonstrated using simulation results.
\end{abstract}

Keywords: Distillation Column, Dynamic Neural Network, Nonlinear $\mathrm{H}_{\infty}$ Control

\section{Introduction}

A distillation column is a strongly nonlinear process with multivariate interactions among outputs and some uncertainty often exists in the system, which renders the analysis and control of a distillation column very difficult $[1,2]$. In practice, the single-point control is commonly used which is sample and easy to tune. However, the consumption of energy in single-point control is large and the product of the other end is not guaranteed. For high purity distillation column, the two-point control or other strategies have been investigated [1,2]. Considering the intrinsic nonlinearity of a distillation column, nonlinear controller has been designed based on the rigorous mathematical model $[3,4]$. Although a nonlinear controller may be effective in simulation, its implementation in practical plants is complex because of the lack of measurement of some key variables. Furthermore, a controller based on the accurate mathematical model may lead to poor performance in case of large perturbation. For this reason, most controllers for distillation columns are synthesized based on the input-output relations such as transfer function or a model obtained by system identification [1,5-8].

Because of its capability to approximate arbitrary nonlinear mapping, neural network has been actively used in nonlinear system identification and control [9-12]. The multilayer feed-forward neural network (MFNN) is one of the most widely used neural networks as a system model in the design of a model-based controller. A dy- namic neural network (DNN) is more suitable to approximate a nonlinear dynamic process. Therefore, dynamic recurrent neural network has been used to learn the input-output relationship of a column and a local optimal controller based on the neural network model was given in [8]. Non-linear adaptive controllers based on MFNN and RBFNN have also been studied $[13,14]$. A recent review [15] for the past 28 years showed that most of the implementations of advanced control like internal model control were based on linear models. Many recently published papers $[16,17]$ on neural control for distillation columns are some extensions of previous research and supported by simulations. Although a neural network can be trained in simulation to approximate any nonlinear process, the control law must be designed to be robust for the modeling error. $\mathrm{H}_{\infty}$ control has been a very popular for robust control of nonlinear system [18-20]. The application of $\mathrm{H}_{\infty}$ control to distillation columns has not been widely discussed.

In this paper, a dynamic neural network is designed to identify the input-output relation of a distillation column online. The training algorithm of the network is derived from a Lyapunov function so that the convergence of weights and the finite bound of the identification error are guaranteed. To cope with model error, a nonlinear $\mathrm{H}_{\infty}$ controller based on the trained network model is employed to enhance the robustness of the control system. Simulation results demonstrate the effectiveness of the online identifier and the control algorithm. 


\section{DNN Based On-Line Identifier of a Binary Distillation Column}

Assume the distillation column is with $(L, V)$ control structure where $L$ and $V$ are reflux flow in condenser and boil up flow in reboiler $(\mathrm{kmol} / \mathrm{min})$ respectively. Then, let $u 1, u 2$ be $L, V$ respectively. The outputs are the light compositions of the top and bottom products of a distillation column.

In some set points, assume the nonlinear input-output relationship of the distillation column can be expressed approximately as below:

$$
\dot{Y}=f(Y)+\left(\begin{array}{ll}
g_{1}(Y) & g_{2}(Y)
\end{array}\right)\left(\begin{array}{l}
u_{1}(t) \\
u_{2}(t)
\end{array}\right)
$$

where $f(Y), g_{i}(Y) \in R^{2 \times 1}, g_{i}(Y)=\left(g_{1 i}(Y) \quad g_{2 i}(Y)\right)^{T}$, and according to some mechanistic knowledge, $g_{11}>0$, $g_{22}<0, g_{12}<0, g_{21}>0$ for any $Y_{i} \in[0,1], i=1,2$.

Assume the outputs $Y_{1}, Y_{2}$ are known, a dynamic neural network based identifier is designed to approximate (1) as below:

$$
\begin{aligned}
\dot{\hat{Y}}= & A \hat{Y}+\hat{f}\left(Y, W_{f}\right)-A Y \\
& +\left(\hat{g}_{1}\left(Y, W_{g_{1}}\right) \hat{g}_{2}\left(Y, W_{g_{2}}\right)\right)\left(\begin{array}{l}
u_{1}(t) \\
u_{2}(t)
\end{array}\right)
\end{aligned}
$$

where $A$ is any stable matrix. $Y$ and $\hat{Y}$ are the output vectors of the column and the identifier respectively. $\hat{f}$ and $\hat{g}_{i}(i=1,2)$ are all MFNN, which are the estimates of $f$ and $g_{i}$ in (1) respectively. The active function of the output-layer of MFNN $\hat{f}$ is linear. The active function of $\hat{g}_{1}$ is a sigmoid function

$k /(1+\exp (-x))$, and the active function of $\hat{g}_{2}$ is $-k /(1+\exp (-x))$, where $k$ is a tunable parameter satisfying $k>0 . W_{f}$ and $W_{g_{i}}$ are the weight matrixes.

From [9], for $\forall \varepsilon>0$ and any continuous function $F(x)$ there exist a three-layer feedforward network satisfing:

$$
\sup _{x}\left\|F(x)-\hat{F}\left(x, W^{*}\right)\right\|<\varepsilon
$$

where $W^{*}$ denotes the optimal weight matrix and $\hat{F}\left(x, W^{*}\right)$ denotes the outputs of the network.

Let $W_{f}^{*}$ and $W_{g_{i}}^{*}$ be the corresponding optimal weight matrix, system (1) can be rewritten as:

$$
\dot{Y}=A Y+\hat{f}\left(Y, W_{f}^{*}\right)-A Y+\sum_{i=1}^{2} \hat{g}_{i}\left(Y, W_{g_{i}}^{*}\right) u_{i}(t)+\xi(Y)
$$

where $\xi(Y)$ is the modeling error of the optimal identifer satisfing:

$$
\sup _{Y \in \Omega_{Y}}\|\xi(Y)\| \leq \bar{\xi}, \quad \Omega_{Y}=\left\{Y \mid 0<Y_{1}, Y_{2}<1\right\}
$$

which can be made as small as possible by adjusting the neural network structure.

Let $\theta$ be an extended column vector of all the ele- ments of a weight matrix $W . \theta^{*}$ denotes the corresponding extended vector of the optimal weight matrix. Then the extended column vector of $W_{f}$ and $W_{g_{i}}$ are $\theta_{f}$ and $\theta_{g_{i}}$. Define identification error as $e=Y-\hat{Y}$ and parameter estimation error as $\tilde{\theta}_{j}=\theta_{j}^{*}-\theta_{j} \quad\left(j=f, g_{i}\right)$. From (2) and (3) we have

$$
\dot{e}=A e+\tilde{f}+\sum_{i=1}^{2} \tilde{g}_{i} u_{i}(t)+\xi(Y)
$$

where

$$
\begin{gathered}
\tilde{f}=\hat{f}\left(Y, \theta_{f}^{*}\right)-\hat{f}\left(Y, \theta_{f}\right)=\frac{\partial \hat{f}}{\partial \theta_{f}} \tilde{\theta}_{f}+O_{\tilde{f}}\left(\left\|\tilde{\theta}_{f}\right\|^{1+\alpha}\right), \\
0<\alpha<1, \\
\tilde{g}_{i}=\hat{g}_{i}\left(Y, \theta_{g_{i}}^{*}\right)-\hat{g}_{i}\left(Y, \theta_{g_{i}}\right)=\frac{\partial \hat{g}_{i}}{\partial \theta_{g_{i}}} \tilde{\theta}_{g_{i}}+O_{\tilde{g}_{i}}\left(\left\|\tilde{\theta}_{g_{i}}\right\|^{1+\alpha}\right)
\end{gathered}
$$

Define

$$
d(t)=O_{\tilde{f}}+\sum_{i=1}^{2} O_{\tilde{g}_{i}} u_{i}(t)+\xi(Y)
$$

Assume:

$$
\sup \|d(t)\| \leq \varepsilon_{f}+\varepsilon_{g} \sup u
$$

where $\varepsilon_{j}(j=f, g)$ is a finite constant.

The updating algorithms of the parameter vectors $\theta_{f}$ and $\theta_{g_{i}}$ are as followings:

$$
\begin{gathered}
\dot{\theta}_{f}=\left(\frac{\partial \hat{f}}{\partial \theta_{f}}\right)^{\tau} P e-k_{f}\|P\| \cdot\|e\| \theta_{f}, \\
\dot{\theta}_{g_{i}}=\left(\frac{\partial \hat{g}_{i}}{\partial \theta_{g_{i}}}\right)^{\tau} P e u_{i}(t)-k_{g_{i}}\|P\| \cdot\|e\| \theta_{g_{i}}
\end{gathered}
$$

where $k_{j}>0, j=f, g_{i}$, and $P$ is the positive defined solution of the following Lyapunov equation:

$$
P A+A^{T} P=-Q
$$

$Q$ is a positive defined matrix and the minimal eigenvalue of $Q$ is $\lambda_{0}$.

Theorem 1: If $u(t)$ is bounded and the weight updating alogrithm is (7), then the identification error $e$ and parameter estimation error $\tilde{\theta}_{j}\left(j=f, g_{i}\right)$ are all ultimately uniformly bounded (UUB)

Proof: Consider the Lyapunov function candidate:

$$
V=\frac{1}{2} e^{\tau} P e+\frac{1}{2}\left(\tilde{\theta}_{f}^{\tau} \tilde{\theta}_{f}+\sum_{i=1}^{2} \tilde{\theta}_{g_{i}}^{\tau} \tilde{\theta}_{g_{i}}\right) .
$$

Differentiating it along (4) and (5) yields

$$
\begin{aligned}
\dot{V} & =-\frac{1}{2} e^{\tau} Q e+e^{\tau} P \frac{\partial \hat{f}}{\partial \theta_{f}} \tilde{\theta}_{f}+e^{\tau} P \sum_{i=1}^{2} \frac{\partial \hat{g}_{i}}{\partial \theta_{g_{i}}} u_{i}(t) \tilde{\theta}_{g_{i}} \\
& +e^{\tau} P d(t)-\dot{\theta}_{f}^{\tau} \tilde{\theta}_{f}-\sum_{i=1}^{2} \dot{\theta}_{g_{i}}^{\tau} \tilde{\theta}_{g_{i}}
\end{aligned}
$$


Substituting (10) into the above equation leads to

$$
\begin{aligned}
\dot{V} & =-\frac{1}{2} e^{\tau} Q e+e^{\tau} P d(t)+\|e\| \cdot\|P\|\left(k_{f} \tilde{\theta}_{f}^{\tau}\left(\theta_{f}^{*}-\tilde{\theta}_{f}\right)\right. \\
& +\sum_{i=1}^{2} k_{g_{i}} \tilde{\theta}_{g_{i}}{ }^{\tau}\left(\theta_{g_{i}}^{*}-\tilde{\theta}_{g_{i}}\right) \\
\dot{V} & \leq-\frac{\lambda_{0}}{2}\|e\|^{2}+\|e\| \cdot\|P\| \cdot\|d\|+\|e\| \cdot\|P\| \cdot\left(-k_{f}\left\|\tilde{\theta}_{f}\right\|^{2}\right. \\
& \left.-\sum_{i=1}^{2} k_{g_{i}}\left\|\tilde{\theta}_{g_{i}}\right\|^{2}+k_{f}\left\|\tilde{\theta}_{f}\right\| \cdot\left\|\theta_{f}^{*}\right\|+\sum_{i=1}^{2} k_{g_{i}}\left\|\tilde{\theta}_{g_{i}}\right\| \cdot\left\|\theta_{g_{i}}^{*}\right\|\right)
\end{aligned}
$$

We can further have:

$$
\begin{aligned}
& \dot{V} \leq-\frac{\lambda_{0}}{2}\|e\|^{2}-\|P\| \cdot\|e\|\left(k_{f}\left(\left\|\tilde{\theta}_{f}\right\|-\frac{1}{2}\left\|\theta_{f}^{*}\right\|\right)^{2}-\frac{k_{f}}{4}\left\|\theta_{f}^{*}\right\|^{2}\right. \\
& \left.+\sum_{i=1}^{2} k_{g_{i}}\left(\left(\left\|\tilde{\theta}_{g_{i}}\right\|-\frac{1}{2}\left\|\theta_{g_{i}}^{*}\right\|\right)^{2}-\frac{1}{4}\left\|\theta_{g_{i}}^{*}\right\|^{2}\right)-\|d\|\right)
\end{aligned}
$$

Assuming $u(t)$ is bounded and combining it with (6), we have

$$
\|d(t)\| \leq \varepsilon_{f}+\varepsilon_{g} \bar{u} \leq \varepsilon_{m} .
$$

So $\varepsilon_{m}$ is bounded.

Define $\varepsilon_{d}=k_{f} \frac{1}{4}\left\|\theta_{f}^{*}\right\|^{2}+\sum_{i=1}^{2} k_{g_{i}} \frac{1}{4}\left\|\theta_{g_{i}}^{*}\right\|^{2}+\varepsilon_{m}$.

It is easy to verify that

if $\|e\| \geq \frac{2}{\lambda_{0}\|P\|} \varepsilon_{d}$

or: $\left\|\tilde{\theta}_{f}\right\| \geq \frac{1}{2}\left\|\theta_{f}^{*}\right\|+\sqrt{\varepsilon_{d} / k_{f}}$,

or: $\left\|\tilde{\theta}_{g_{i}}\right\| \geq \frac{1}{2}\left\|\theta_{g_{i}}^{*}\right\|+\sqrt{\varepsilon_{d} / k_{g_{i}}}$

then $\dot{V}<0$.

For $\theta_{i}^{*}$ being bounded, when $u_{i}(t) \in L_{\infty}$, we have $\varepsilon_{d} \in L_{\infty}$. According to Barbalat's theorem [21], it can be seen that:

$$
e, \tilde{\theta}_{f}, \tilde{\theta}_{g_{i}} \in L_{\infty} .
$$

Considering (5), we further have $\dot{e} \in L_{\infty}$.

\section{Nonlinear $\mathbf{H}_{\infty}$ Control of the Binary Distillation Column}

\subsection{Nonlinear $\mathbf{H}_{\infty}$ Control}

Consider an affine nonlinear controller system

$$
\begin{aligned}
\dot{x} & =\bar{f}(x)+\bar{g}_{1}(x) \omega+\bar{g}_{2}(x) u \\
y & =h(x)+k(x) u
\end{aligned}
$$

where $x \in R^{n}, u \in R^{m}, \omega \in R^{s}, y \in R^{p} ; \bar{f}(x), \bar{g}_{1}(x)$, $\bar{g}_{2}(x), h(x)$ and $k(x)$ are smooth functions; $u$ is control input $\omega$ is uncertainty noise and $\bar{f}(0)=0, h(0)=0$.

For system (8), the design objective of nonlinear $\mathrm{H}_{\infty}$ control is to make the following conditions guaranteed $[19,20]$.

1) The closed-loop system is asymptotically stable; and,

2) for a given $\gamma>0$, the following inequality holds

$$
\int_{0}^{t}\|y(\tau)\|^{2} \mathrm{~d} \tau \leq \gamma^{2} \int_{0}^{t}\|\omega(\tau)\|^{2} \mathrm{~d} \tau \quad \forall t \geq 0 .
$$

According to [19], defining $V_{x}=\frac{\partial V}{\partial x}$ for $\forall x \in R^{n}$ and $R(x)=k^{T}(x) k(x)$, we have:

Proposition: If $R(x)$ is nonsingular, nonlinear $\mathrm{H}_{\infty}$ control law is expressed as

$$
u=-R^{-1}(x)\left(\frac{1}{2} \bar{g}_{2}^{\tau}(x) V_{x}^{\tau}+k^{\tau}(x) h(x)\right)
$$

where $V(x)(\mathrm{V}(0)=0)$ satisfies

$$
V_{x} \hat{\bar{f}}(x)+\hat{h}^{\tau}(x) \hat{h}(x)+\frac{1}{4} V_{x} \hat{R}(x) V_{x}^{\tau} \leq 0
$$

where

$$
\begin{gathered}
\hat{\bar{f}}(x)=\bar{f}(x)-\bar{g}_{2}(x) R^{-1}(x) k^{\tau}(x) h(x) \\
\hat{h}(x)=\left(I-k(x) R^{-1}(x) k^{\tau}(x)\right) h(x) \\
\hat{R}(x)=\frac{1}{\gamma^{2}} \bar{g}_{1}(x) \bar{g}_{1}^{\tau}(x)-\bar{g}_{2}(x) R^{-1}(x) \bar{g}_{2}^{\tau} .
\end{gathered}
$$

\subsection{Nonlinear $H_{\infty}$ Control of the Binary Distillation Column}

Considering the existence of the modeling error, system (3) can be rewritten as:

$$
\dot{Y}=A Y+\hat{f}-A Y+\left(\begin{array}{ll}
\hat{g}_{1} & \hat{g}_{2}
\end{array}\right) u(t)+\omega
$$

where $\omega$ is the uncertainty variable denoting the modeling error.

Assuming the desired tracking trajectory is $\dot{Y}_{d}=A Y_{d}+s$, where $S$ is a reference variable.

$$
\text { Define }\left(\begin{array}{c}
x_{1} \\
x_{2}
\end{array}\right)=Y-Y_{d}=\left(\begin{array}{c}
Y_{1}-Y_{1 d} \\
Y_{2}-Y_{2 d}
\end{array}\right) \text {. }
$$

From (10), the tracking error equation can be derived as below:

$$
\dot{x}=a(x)+A x+G_{1} \omega+G_{2} u
$$

where

$$
a(x)=\hat{f}-A Y-s, G_{1}=\left(\begin{array}{ll}
1 & 0 \\
0 & 1
\end{array}\right), G_{2}=\left(\begin{array}{ll}
\hat{g}_{11} & \hat{g}_{12} \\
\hat{g}_{21} & \hat{g}_{22}
\end{array}\right) .
$$


For the binary distillation column, $G_{2}(Y)$ is invertible. If the penalty variables are chosen to be

$$
z=h(x)+k(x) u=a(x)+G_{2} u .
$$

Substituting (9) into (12), we have:

$$
\begin{aligned}
& u=-R^{-1}(x)\left[G_{2}^{T}(x) P x+k^{T}(x) h(x)\right] \\
& =-G_{2}^{-1}(P x+a(x))
\end{aligned}
$$

where $P$ is the positive defined solution of the following Riccati inequality:

$$
P A+A^{T} P+P\left(\frac{1}{\gamma^{2}}-1\right) P \leq 0 .
$$

Remark: $P$ can be obtained by solving a Riccati equation as below:

$$
P A+A^{T} P+P\left(\frac{1}{\gamma^{2}}-1\right) P+C^{T} C=0
$$

where $[A, C]$ is observable.

\section{Simulation}

After The binary distillation model used in the simulation is the same as the one developed by [2]. The nominal values of outputs are $Y_{10}=0.98(\mathrm{~mol} \%)$ and $Y_{20}=0.02(\mathrm{~mol} \%)$. The nominal values of reflux flow and boil-up flow are $L=2.28625(\mathrm{kmol} / \mathrm{min})$ and $V=2.78625(\mathrm{kmol} / \mathrm{min})$. The feed flow and feed composition are $F=1(\mathrm{kmol} / \mathrm{min})$ and $Z_{f}=0.5(\mathrm{~mol} \%)$.

The distillation column is controlled using the control strategy (13) developed in this paper. The tacking property and the disturbance attenuation property of the control system are demonstrated through simulation. To make the transient response be more elegant, the dynamic neural network based identifier is trained for some time offline.

In the simulation, we choose

$$
A=\left(\begin{array}{cc}
-9 & 0 \\
0 & -9
\end{array}\right), \quad C=\left(\begin{array}{ll}
1 & 0 \\
0 & 1
\end{array}\right), \gamma=1.1
$$

and the positive definite $P$ we obtained is

$$
P=\left(\begin{array}{cc}
0.0555 & 0 \\
0 & 0.0555
\end{array}\right) \text {. }
$$

1) Servo properties

Let the setpoint of the top light component change from nominal value 0.98 to 0.995 and the bottom composition remain nominal value 0.02 . The augmentations of system output are demonstrated in Figure 1. The curves of the control inputs are in Figure 2.

2) Robustness

The responses of the closed-loop system are illus-

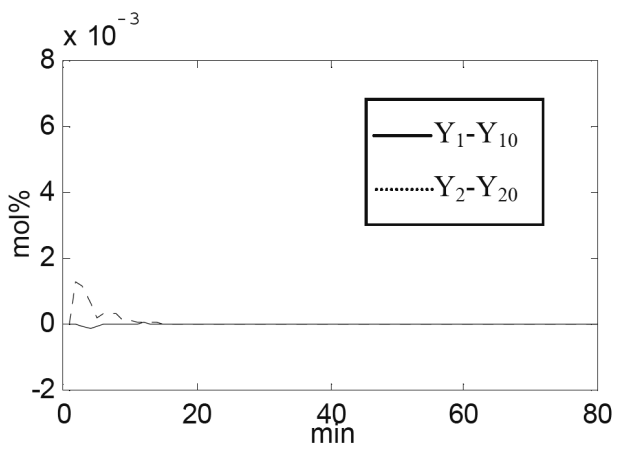

Figure 1. The augmentations of outputs.

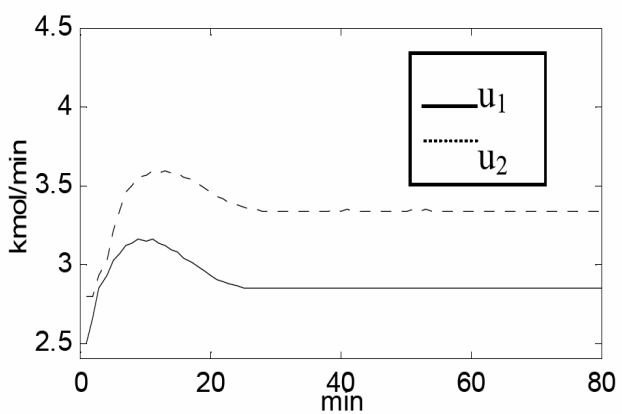

Figure 2. The curves of control inputs.

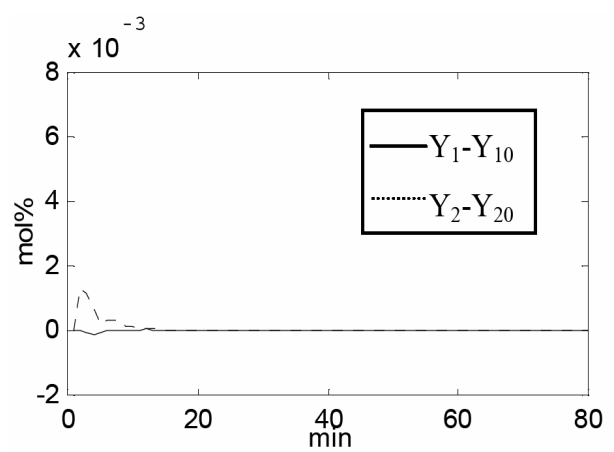

Figure 3. The augmentations of outputs (Feed increases $10 \%)$.

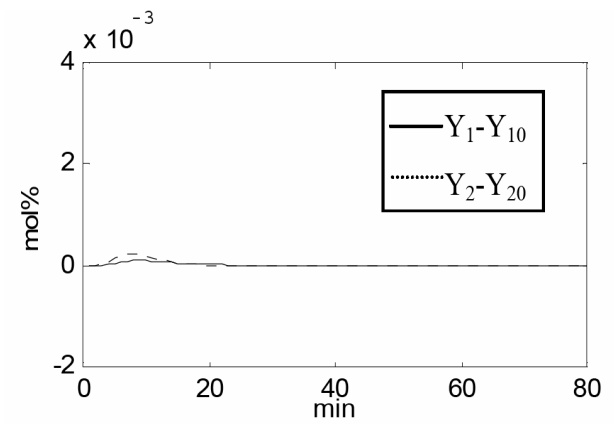

Figure 4. The augmentations of outputs (Feed composition increases $10 \%$ ).

trated in Figures 3 and $\mathbf{4}$ when feed flow $F$ and feed composition $Z_{f}$ increase $10 \%$ respectively. 


\section{Conclusions}

A dynamic neural network based online nonlinear identifier for a binary distillation column is designed. The learning algorithm of the network weights is established in detail, which can guarantee the boundedness of the identification error. To deal with the modeling error, a nonlinear $\mathrm{H}_{\infty}$ controller based on the identifier is given by choosing the penalty variables for the column. The effectiveness of the proposed strategy is demonstrated in simulation results. The algorithm developed in this paper can be applied to other chemical processes as well.

\section{Acknowledgements}

The author thanks the reviewers for very helpful comments. Views expressed in this paper are the author's professional opinions and do not necessarily represent the official position of the US Food and Drug Administration.

\section{References}

[1] T. J. McAvoy and Y. H. Wang, "Survey of Recent Distillation Control Results," ISA Transactions, Vol. 25, No. 1, 1986, pp. 5-21.

[2] S. Skogestad and M. Morari, "Understanding the Dynamic Behavior of Distillation Columns," Industrial \& Engineering Chemistry Research, Vol. 27, No. 10, 1988, pp. 1848-1862. doi:10.1021/ie00082a018

[3] J. Lévine and P. Rouchon, "Quality Control of Binary Distillation Columns via Nonlinear Aggregated Models," Automatica, Vol. 27, No. 3, 1991, pp. 463-480. doi:10.1016/0005-1098(91)90104-A

[4] F. Viel, E. Busvelle and J. P. Gauthier. "A Stable Control Structure for Binary Distillation Columns," International Journal of Control, Vol. 67, No. 4, 1997, pp. 475-505. doi:10.1080/002071797224036

[5] N. F. Jerome and W. H. Ray, "High-Performance Multivariable Control Strategies for Systems Having Time Delays," AIChE, Vol. 32, No. 6, 1986, pp. 914-931. doi:10.1002/aic.690320603

[6] S. Skogestad, M. Morari and J. C. Doyle, "Robust Control of Ill-Conditioned Plants: High Purity Distillation," IEEE Transactions on Automatic Control, Vol. 33, No. 12, 1988, pp. 1092-1105. doi:10.1109/9.14431

[7] J. Savkovic, "Neural Net Controller by Inverse Modeling for a Distillation Plant," Computers \& Chemical Engineering, Vol. 20, No. S2, 1996, pp. S925-S930. doi:10.1016/0098-1354(96)00162-7

[8] W. Yu, S. Alexander and J. A. Poznykz, "Neuro Control for Multicomponent Distillation Column," 1999 IFAC
World Congress, Beijing, 5-9 July 1999, pp. 379-383.

[9] G. Cybenko, "Approximation by Superpositions of Sigmoidal Function," Mathematics of Control, Signals, and Systems, Vol. 5, No. 4, 1989, pp. 303-314. doi:10.1007/BF02551274

[10] K. Hunt, J. D. Sbarbaro, R. Zbikowshi and P. J. Gawthrop, "Neural Networks for Control Systems-A Survey," Automatica, Vol. 28, No. 6, 1992, pp. 1083-1112. doi:10.1016/0005-1098(92)90053-I

[11] M. J. Wills, G. A. Montague, C. Di Massimo, M. T. Tham and A. J. Morris, "Artificial Neural Networks in Process Esitmation and Control," Automatica, Vol. 28, No. 6, 1992, pp. 1181-1187. doi:10.1016/0005-1098(92)90059-O

[12] P. Turner, G. Montagueand J. Morris, "Dynamic Neural Networks in Nonlinear Predictive Control," Computers \& Chemical Engineering, Vol. 20, No. S2, 1996, pp. S937S942. doi:10.1016/0098-1354(96)00164-0

[13] S. Li and F. Li, "Dynamic Neural Network Based Nonlinear Adaptive Control for a Distillation Column," Proceedings of the 3rd World Congress on Intelligent Control and Automation, Hefei, June 28-July 2 2000, pp. 3087-3091.

[14] S. R. Li, H. T. Shi and F. Li. "RBFNN Based Direct Adaptive Control of MIMO Nonlinear System and Its Application to a Distillation Column," Proceedings of World Conference of Intelligent Control and Automation, Shanghai, 10-14 June 2002, pp. 2896-2900.

[15] D. Muhammad, Z. Ahmad and N. Aziz, "Implementation of Internal Model Control (IMC) in Continuous Distillation Column," Proceedings of the 5th International Symposium on Design, Operation and Control of Chemical Processes, Singapore, 16 February 2010, pp. 812-821.

[16] H. E. Tonnang and A. Olatunbosun, "Neural Network Controller for a Crude Oil Distillation Column," Journal of Engineering and Applied Sciences, Vol. 5, No. 6, 2010 , pp. 74-82.

[17] M. Ławryńczuk, "Explicit Nonlinear Predictive Control of a Distillation Column Based on Neural Models," Chemical Engineering \& Technology, Vol. 32, No. 10, 2011, pp. $1578-1587$.

[18] S. R. Li, "Nonlinear $\mathrm{H}_{\infty}$ Controller Design for a Class of Nonlinear Control Systems," Proceedings of 23rd IEEE Industrial Electronics, Control and Instrumentation, New Orleans, 9-14 November 1997, pp. 291-294.

[19] A. Isidori, "Nonlinear Control Systems," 2nd Edition, Springer-Verlag, New York, 1989.

[20] A. Isidori and A. Astolfi, "Disturbance Attenuation and $\mathrm{H}_{\infty}$ Control via Measurement Feedback in Nonlinear Systems," IEEE Transactions on Automatic Control, Vol. 37, No. 9, 1992, pp. 1283-1293. doi:10.1109/9.159566

[21] V. M. Popov, "Hyperstability of Control Systems," 2nd Edition, Springer-Verlag, New York, 1973. 